\title{
PHYSIOTHERAPY MANAGEMENT OF MARATHON MUSCULO-SKELETAL CASUALTIES
}

\author{
Rose MacDONALD, BA, MCSP \\ Superintendent Physiotherapist, Sports Injury Clinic, \\ Crystal Palace National Sports Centre, LONDON SE 19
}

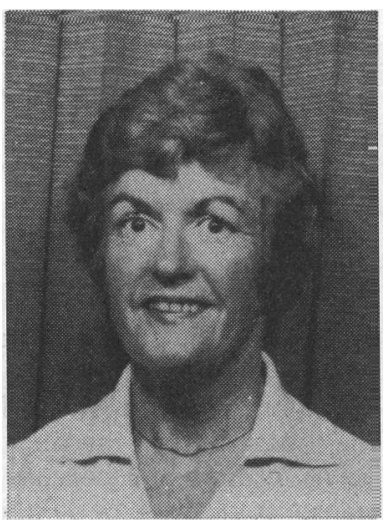

Rose P. Macolonald
Physiotherapists with experience in sports injuries are an essential part of the marathon medical team as they are familiar with the injuries suffered by the long distance runners and are thus able to make a rapid assessment of the injury on the marathon day and treat accordingly. The long distance runner is liable to suffer from the whole gamut of injuries to the lower limb including muscle and tendon strains, joint sprains, trauma to the knee, ankle and foot, ilio-tibial band syndrome and compartment syndromes, knee cap pain, plantar fasciitis, heel spurs, stress fractures and musculoskeletal peritendinitis of the Achilles tendon.

Not all these injuries may be seen at a marathon, but the most common complaints treated at the last four London Marathons were:-

Muscle cramp

Muscle and tendon strains

Trauma to the knee, ankle and foot joints

Stress fractures - shin splints

\section{MUSCLE CRAMP}

This occurs during or immediately after exercise. It is thought to be due to accumulation of waste products from prolonged intensive work. Dehydration is also thought to be a causative factor. Muscle cramp must be differentiated from muscle strain - and a correct diagnosis made before treatment is given. With cramp, the muscle becomes hard and rigid - often while being examined - and is extremely painful. The limb becomes rigid and the runner becomes very distressed. Pain will be decreased when the muscle is stretched to its limit and held, while massaging the belly of the muscle until relaxation of the fibres takes place. Fluid, etc., given to the patient at this time may distract the patient and further help relaxation. Cramp occurs mainly in the calf and hamstring muscles, therefore, getting the runner up and walking as soon as possible will give relief, and the runner is advised to drink plenty of fluid during long runs, at least $8 \mathrm{oz}(150 \mathrm{ml})$ every $20 \mathrm{mins}$ and at every station during a marathon.

\section{MUSCLE AND TENDON STRAINS}

A strain is a tear of a muscle, a tendon or the junction of muscle and tendon. The most common areas for strains are the calf muscles, hamstrings, quadriceps, groin and lower back. The reasons for strained muscles are varied but frequently they are linked to an improper or complete lack of warm-up which should include a stretching regime to increase flexibility in those muscles that are susceptible to strain and tend to tighten up during running. When muscle suffers a first or second degree strain it goes into protective spasm - not to be confused with cramp. The runner usually knows if he has 'torn a muscle' and will tell you so. A cramp will be eased by stretching but a 'torn muscle' will be extremely painful on stretch as further tearing takes place. A muscle tear usually has some swelling, is tender to palpate, and if severe enough, a defect. Immediate application of ice will reduce pain and spasm. Compress the injured site with a pad and apply an elastic bandage or tubigrip to support the whole muscle.

Advice for prevention includes a proper warm-up and warm-down routine and the patient should be shown how to stretch the injured muscle gently under ice until he sees a doctor and physiotherapist for further treatment and rehabilitation.

\section{JOINT SPRAINS}

Trauma to the knee, ankle, foot and sometimes hip are commonly seen. This may be due to repeated stress and fatigue, or because the muscles around the joints are weak and the gait is affected. Poor foot function places stress on other structures and because of this could be the prime cause of joint trauma. Full assessment of joints is not possible at a marathon but immediate first aid must be given to alleviate pain and anxiety. Putting an ice pack on the joint relieves pain and muscle spasm and if there is swelling, compression with an elastic wrap or Tubigrip and elevation of the joint is needed. In the case of an ankle sprain, taping with rigid zinc 
oxide may be necessary, and crutches or transport if the runner is unable to bear weight should be arranged. $A$ visit to the GP for further assessment, and to physiotherapy for rehabilitation is recommended.

\section{STRESS FRACTURES}

Exercise-induced stress fractures are becoming more common because of the current popularity of fitness activities, especially running. Of all the exercise syndromes the stress fracture is the most crippling and necessitates stopping running altogether for 3-8 weeks. Common sites for stress fractures are the metatarsals, the lower third of the tibia and fibula, the femur and the pelvis, presumably because these areas absorb the forces transmitted to the body during running.

A simple finger test helps to diagnose a stress fracture - usually it will hurt if pressed from both above, and below the site by the finger. This injury is commonly associated with the inexperienced athlete who attempts too much too soon, or with the accomplished runner who demands too much of his well trained body. Any dramatic increase in mileage or speedwork will overload the lower extremity, the tibia being most prone to injury. A "crescendo effect" history of pain will suggest the likelihood of a stress fracture, pain is present initially only during running but will progress to discomfort in normal daily walking and standing. At that time the area is usually warm, mildly swollen and tender to touch continued activity may lead to a complete fracture. Applying ice to the tender area for 10-15 minutes relieves pain, support to the bone with tape and advice for a visit to the GP for possible X-ray and bone scan is indicated.

\section{SHIN SPLINTS}

A catch-all term for any pain in the shin, and may be differentiated from a stress fracture by palpation and history. It is a soft tissue condition as opposed to bony tenderness. Causes are varied and include repeated running on hard surfaces, poor support from shoes, poor range of motion at the anterior and posterior aspects of the leg. Ice or ice massage to the tender area relieves pain, and putting both feet in a basin of cold water is also helpful. The shins may be supported with a double tubigrip from. toes to knee. Advising the patient about good shock absorbing shoes, conditioning exercises to stretch and strengthen the muscle at the front of the lower leg, and exercises to improve ankle flexibility all help. Alternative training surfaces can also help to alleviate this condition. If a bio-mechanical problem of the foot is suspected then strapping or orthotics may be necessary.

\section{BLISTERS}

Blisters however small can cause a considerable amount of pain and discomfort and must be dealt with immediately. Should the irritation penetrate to the dermal layer of the skin the result could be a blood-filled blister which is more likely to become infected. If the blister is in a pressure area and interferes with walking and running, piercing at the side with a sterile needle and allowing the fluid to drain out, cleansing well, the use of an antiseptic ointment and dressing with second skin and adhesive are the best proceedings. Apply a doughnut cut from stick-on-padding, or felt, or mole skin, to relieve pressure and prevent re-filling. When the skin of a blister has been torn, extra precautions must be taken to avoid infection by cleaning the blister and surrounding area with soap and water or antiseptic tissue. With sterile scissors the blister is cut about halfway round its perimeter, and antiseptic ointment is applied to the exposed area laying the flap back over the treated area, then second skin and a dressing. Advice to the runner on blister prevention includes:

1. Well fitting shoes - not too loose (allowing the foot to slide around) or too small (squeezing the foot and producing pressure in tight areas).

2. Gradually break in new shoes to fit the form of the foot.

3. Wear two pairs of socks to cut down friction between skin and shoes. The inside pair inside out and the second pair normally.

4. Foot power in the inside sock helps to cut down friction.

5. Treat the areas prone to blister with stick-on-padding or zinc oxide tape applied directly to the skin.

6. Hot spots are pre-blister sites and ice should be applied for ten minutes after each run until the spot is gone.

\section{CHAFING OF THIGHS}

This happens to those whose thighs rub together while running especially when wet, due to sweat or weather. It also happens to those who wear new shorts on the day, or shorts with binding around the legs. The lesions must be cleansed and dressed with second skin and tape. This condition may be prevented by wearing old comfortable shorts, and if the thighs rub, applying zinc oxide tape or adhesive knit to the area where friction occurs. Cleansing the area and applying second skin which is cool and soothing and adhesive knit or Hypafix (which allows healing to take place as it is permeable to air) are sensible steps to take in preventing full skin thickness ulceration.

\section{BLEEDING NIPPLES}

There is no need for this condition to occur if runners wear comfortable vests and if women wear supporting brassieres. Tape over the nipple will prevent friction against a tight vest. Stretch fabric plasters will stick on and stay on better than plastic plasters. The area should be cleansed with soap and water or antiseptic solution, then second skin and adhesive knit as above should be applied. 


\section{PRACTICAL TIPS FOR THE PHYSIOTHERAPIST}

When contacting the organisers before the day of the marathon it is useful to check who will arrange for cots, couches, chairs, blankets, etc. to be available and to ensure that the physiotherapists will be working close to the finish. Checking the type of course, single or double loop for physiotherapist placement, and that hot and cold water, sponges, heating (if cold weather), hoses (if hot weather), and toilets are at hand is vital. If the recovery area is a tent there must be dry floor covering. Arrangements regarding assignment of patient type to the various treatment areas may be carried out by the St. Johns/Red Cross personnel if present.

1. Organise your team well before the event and brief them on duty, location, rota, etc.

2. One physiotherapist per thousand runners will be needed at the recovery area. If placing physiotherapists at the half-way mark or at $\mathbf{1 7}$ miles, more will be needed as this is a troublesome area, advice must be given here to the injured runner whether to continue or not.

3. Clear identification of medical personnel is a must.

4. Be prepared to work as a team with doctors, first aid personnel and podiatrists.

5. If there are no podiatrists on the medical team, be prepared to deal with blisters and sub-ungual haematomas, etc.

6. Use some form of treatment record card for future statistics - these may be provided by the doctor in charge.

7. Well before the marathon date solicit medical supplies, e.g. second skin, tape, plasters, crepe bandages, adhesive knit, hypafix, tubigrip, creams, lotions and oils for massage.
8. Make sure that ice will be available - small ice coolers filled with ice towel packs are easy to carry around.

9. The majority of runners needing attention will need stretching and massage.

10. The bulk of the casualties start arriving about 3 hours after the start and continue arriving for a further 2-3 hours.

\section{TIPS ON INJURY PREVENTION FOR THE MARATHON RUNNER}

Stress the necessity for a good warm-up and warmdown.

- advise on the importance of stretching all the muscles especially the calves and hamstrings before and after running (these two muscle groups are not put through the full range of motion when running therefore the hamstrings and Achilles tendons become tight and prone to injury if not stretched regularly).

- advise on running surface ... a mixture of hard and soft is ideal when training.

- advise on good shoes and the need for shock absorbing inserts if necessary.

- advise on a balanced progressive increase in endurance work ... gradualness is the key word when training.

A good rule of thumb for increasing distance is to add $10 \%$ of the present mileage per week to the running schedule. This should allow an increase of mileage ando a minimum of stress and strain to the body.

Be well prepared and well trained for the event.

After the event... a hot bath as soon as possible, gentle stretching and jogging over the next few days and a gradual return to running. 